\title{
Power Control Strategy for Unity Power Factor
}

\author{
K. Jezernik \\ University of Maribor \\ Faculty of Electrical Engineering and Computer Science \\ Smetanova 17, SI-2000 Maribor, Slovenia \\ Phone/Fax number:+386-2-220 73 00/+386-2-220 73 15, e-mail: karel.jezernik@uni-mb.si
}

\begin{abstract}
With the introduction of recent standards on limitation of harmonic pollution of electrical power distribution system, three-phase PWM converters are considered as prime candidates to interface high-power electronic equipment to power supply lines. In these applications, converters can provide input currents without distortion and with the unity power factor. In this paper, a unified discrete event model is given for power electronic circuits, based on a hybrid system theory. Based on this model, FPGA switching control strategy for a three phase converter is developed. The functionality of a three phase converter is discussed from a discrete-event point of view. Recently developed hybrid based approach for modeling of discrete event systems is applied for modeling, simulations and implementation of a power factor control, protection and steering functionality three-phase converter. A DSP / FPGA based digital control platform for converter system, built in the laboratory, is presented and discussed.
\end{abstract}

\section{Key words}

Unity power factor, Hybrid switching control, Three phase converter, FPGA.

\section{Introduction}

The conventional ac/dc power converters that are connected to the line through full wave rectifiers draw a sinusoidal input ac current. Harmonic content in a current waveform that flows through the impedances in the electric utility distribution system can create harmonic voltages. These harmonics distort the local voltage waveform, which might potentially interfere with other electrical equipment connected to the same electrical service. Also, a distorted ac input current waveform prohibits the extraction of the maximum possible real power from the utility service. Unity power factor converters employ active wave shaping of the ac input current to ensure a sinusoidal current slope, while they deliver a constant dc output voltage [1].

Typical control schemes employed in state-of-the-art unity power factor converters have fairly low bandwidth in order to limit the impact of output voltage ripple on input power factor. In such controllers, disturbances are attended by control actions taken at most in the order of twice per line cycle [2].

The fast controller, presented here, executes control action at a much faster rate. As a result, the fast controller achieves a fast response time to disturbances. A key feature of our controller is its ability to reject feedback of the ripple on the dc bus capacitor by actively canceling it, so that high bandwidth control can be maintained without distorting the input ac current during steady state operation [3].

Special attention is paid to the new current control principle where traditional scheme, which consists of discrete-time current controller and pulse-width modulator, is replaced with a new discrete-event current controller [4]. The key idea, used for the event-driven current control approach, is to evaluate the transistor switching pattern directly from the phase current errors. The idea originates from the hysteresis current control principle. In this paper, the initial idea is further developed with the introduction of switching pattern sequences for the switching frequency reduction. Additionally, systematic design methodology is introduced for the design of multivariable sequential discrete event-driven systems (DES) control principle. Same formalism is also used for design of functionality, which provides the management and the protection of the converter.

Lyapunov theory has for long time been an important tool in linear as well as nonlinear control. However, its use within the nonlinear control has been changed by the difficulties to find the Lyapunov function for a given system. If one can be found, the system is known to be stable, but the task of finding such function has often been left to the imagination and experience of the designer [5].

The aim of this paper is the design of a control law for a three-phase boost rectifier to achieve good power and grid current control in steady state and transient operating conditions. The feedback system is globally asymptotically stable in the sense of the Lyapunov stability theory. Therefore we are interested in an extension of the Lyapunov function concept. This concept used such a scalar function, which contains a logical discontinuous input switching function that penalizes the grid current control error, which enhances the stability.

The paper starts with a brief discussion of hybrid modeling for the discrete event-driven systems. The case study starts with the three-phase boost converter current controller design and the design of inverter steering and protection functions. Evolved models are checked with simulations using MATLAB / Simulink and are experimentally confirmed. During the experiment, mapping of the obtained models into the FPGA executable code is presented [6]. The findings and the comments of the presented approach are discussed in the conclusion. 


\section{System Analysis and Control}

\section{A. Hybrid Switching Control}

The field of power electronic poses challenging control problems that cannot be treated in a complete manner using traditional modeling and controller design approaches. The main difficulty arises from the hybrid nature of these systems due to the presence of semiconductor switches that include different modes of operation and operate with high switching frequency. The computational power, available today, and the recent theoretical advances in the control of hybrid systems allow one to tackle these problems in a novel way that improves the performance of the system and is systematic and implemental.

Given here are a unified model of power electronic circuits, which can be described as a network of electrical components selected from the following three groups: ideal voltage or current sources, linear elements (e.g. resistors, capacitors, inductors, transformers), and nonlinear switching elements (e.g. IGBT, power MOSFET, power diode). In the following analysis, the behavior of a switch is idealized as having two discrete states: open or close. In every discrete state, power electronic frame system behaves corresponding to the continuous dynamic.

The basic principle considered in power electronic circuits is the switching control. In switching control, one built bank of alternative candidate voltages depends on the configuration of switching elements in power electronic circuits. By VSI, the switching is orchestrated by a specially designed decision logic, that uses the measurements of continuous state variables, currents, to asses the performance of the candidate voltage input vector $\boldsymbol{V}_{i}$ currently in use, and also the potential performance of alternative voltage input vectors $\boldsymbol{V}_{i-1}$, $\boldsymbol{V}_{i+1}$. Fig. 1 shows the basic architecture employed by switching control. In this figure $\boldsymbol{u}_{s}\left(\boldsymbol{V}_{i}\right)$ represents the discontinuous control input voltage vector, $\boldsymbol{e}_{s}$ the grid voltage vector and $\boldsymbol{i}_{s}$ the grid input current. The dashed box is a conceptual representation of a switching controller. The top element in Fig. 1 is the selector that controls the switches, or more precisely that generates the switching control input vector $\boldsymbol{S}\left(S_{1}, S_{2}, S_{3}\right)$. The selector is called the supervisor and its purpose is to monitor the signals that can be measured $\left(\boldsymbol{u}_{S}\left(\boldsymbol{V}_{i}\right)\right.$, current control error $\Delta \boldsymbol{i}_{s}$ ) and decide, at each instant of time, which candidate voltage vector $V_{i}$ should be put in the feedback loop with the process. In the supervisory control, the selector combines continuous dynamic with discrete logic and is therefore a hybrid system, i.e. discontinuous input $\boldsymbol{u}_{s}\left(\boldsymbol{V}_{i}\right)$ and feedback continuous input current $i_{s}$. The modeling of such systems has received considerable attention in the control and computer science literature in the last few years. Power electronics and motion control system is rather a new approach with a lack of applications in a hybrid based control approach.

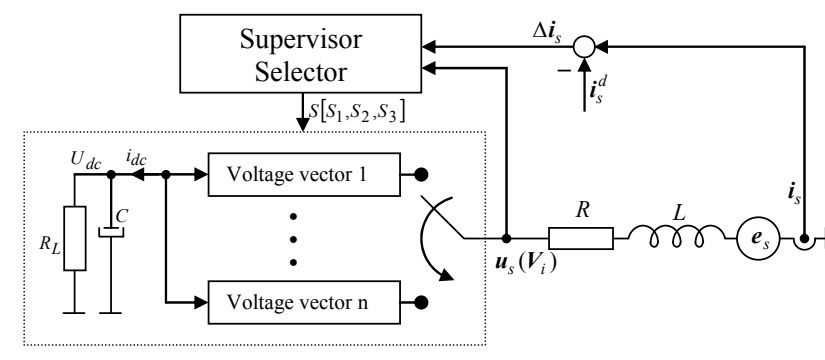

Fig. 1. Switching supervisory control

The proposed system configuration is shown in Fig. 2. The main circuit consists of a bridge rectifier made up of six power transistors with antiparallel diodes, which is connected to the three-phase supply, through an inductor $L$. A load and a capacitor $C$ are connected to the dc side of the converter. The inductor $L$ performs the voltage boost operation in combination with the capacitor $C$ and at the same time acts as a low pass filter for the ac line current.

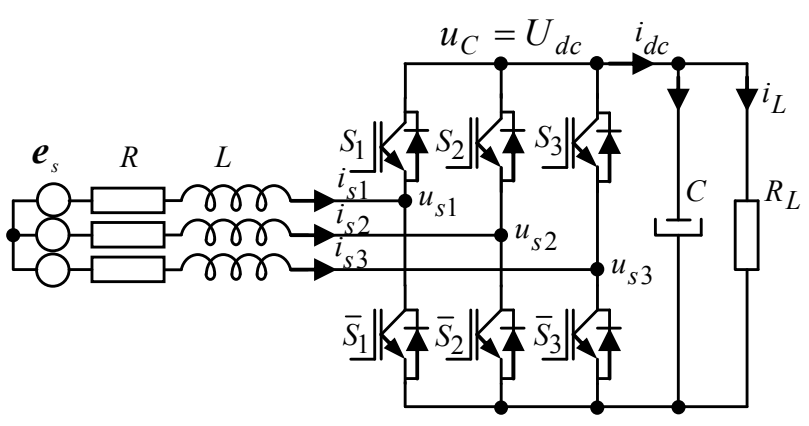

Fig. 2. System configuration

The dynamics of a three-phase boost rectifier in the three-phase reference frame for ac current control system is described as:

$$
\begin{aligned}
& L \frac{d i_{j}}{d t}+R i_{j}=e_{s j}-u_{s j}, \\
& u_{s j}=\left(d_{j}-\left(d_{1}+d_{2}+d_{3}\right) / 3\right) u_{C}
\end{aligned} \quad j=1,2,3
$$

and for output voltage control system as:

$$
C \frac{d u_{C}}{d t}=i_{d c}-i_{L}=i_{1} d_{1}+i_{2} d_{2}+i_{3} d_{3}-\frac{u_{C}}{R_{L}}
$$

where $d_{j}$ is duty ratio.

The main problem in design of the control law of the boost rectifier is occurrence of control input function in both ac current and dc voltage control systems of the converter. Both input functions depend on the same duty ratio $d_{j}$ by different dynamics behavior of ac input and $\mathrm{dc}$ output of the boost rectifier. Input grid voltages and currents should be sinusoidal shaped without a phase delay between the voltage and the current and output $\mathrm{dc}$ voltage should be a constant value by change of voltage dependent current and parameters of the converter [7]. The considered control problem is the tracking of a three phase current reference signal. After defining current control error $\Delta \boldsymbol{i}_{s}=\boldsymbol{i}_{s}-\boldsymbol{i}_{s}^{d}$, rewritten in error form becomes

$$
L \frac{d}{d t} \Delta \boldsymbol{i}_{s}+R \Delta \boldsymbol{i}_{s}=\boldsymbol{e}_{s}-\boldsymbol{u}_{s}\left(\boldsymbol{V}_{i}\right)
$$


which collects all the disturbances (exogenous and endogenous) action on the system.

The basic principle of the current control is to manipulate the input voltage vectors so that the desired current of power electronic circuits is produced. This is achieved by choosing a converter switch combination $S_{i}$ that drives the grid current vector by directly applying the appropriate voltages $\boldsymbol{u}_{S}\left(\boldsymbol{V}_{i}\right)$. The switch positions of the three-phase converter are described using the logical variables $V_{\mathrm{i}}$, dependent if switch $S_{\mathrm{i}}$ is $\mathrm{ON}$ or OFF. Each variable corresponds to one phase of the converter (Fig. 2 ). Three-phase converter can produce $2^{3}$ voltage vector combinations; two of them are zero vectors and 6 active vectors, Fig. 3.

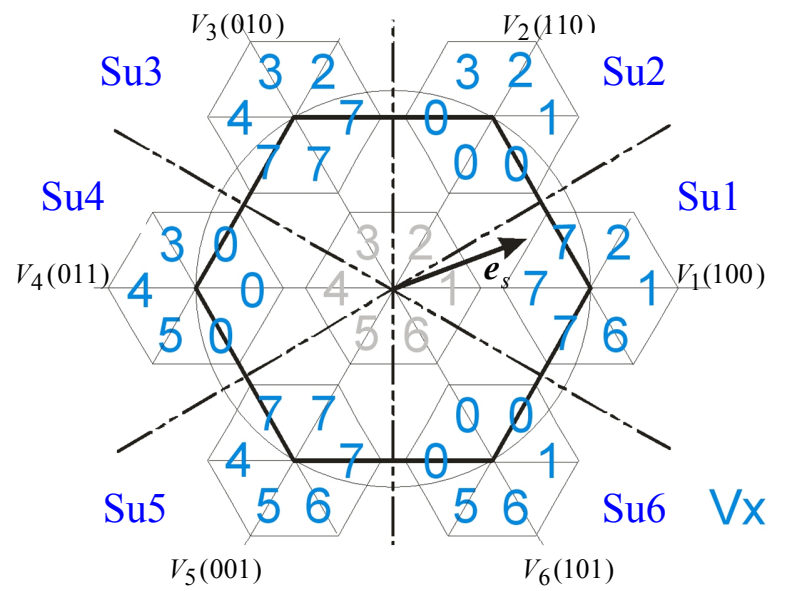

Fig. 3. Input voltage vector $\boldsymbol{u}_{s}$ sector allocation spaces

The energy flow between the input and the output side of the three-phase converter is controlled by switching matrix. By introducing the binary variables $S_{i}$ that are "1" if particular switch $S_{i}$ is On and "0" if switch $S_{i}$ is OFF $(\mathrm{i}=1,2,3, \ldots, 6)$ the behavior of the switching matrix can be described by the three dimensional vector $\boldsymbol{u}_{s}=U_{d c} \boldsymbol{L} \boldsymbol{S}_{i}$, where matrix $L$ and vector $\boldsymbol{S}\left(S_{1}, S_{2}, S_{3}\right)$ are defined as [8]

$$
\begin{aligned}
\mathbf{L} & =\left[\begin{array}{cccccc}
1 & 0 & 0 & -1 & 0 & 0 \\
0 & 1 & 0 & 0 & -1 & 0 \\
0 & 0 & 1 & 0 & 0 & -1
\end{array}\right] \\
\mathbf{S}^{T} & =\left[\begin{array}{llllll}
S_{1} & S_{2} & S_{3} & \bar{S}_{1} & \bar{S}_{2} & \bar{S}_{3}
\end{array}\right]
\end{aligned}
$$

Relation (4) is true for the switching matrix $L$ that is depicted in Fig. 2. It essentially shows that this particular switching matrix is able to generate three independent control actions denoted as the components $S_{1}, S_{2}$ and $S_{3}$ of the control vector $\boldsymbol{u}_{s}\left(\boldsymbol{V}_{i}\right)=U_{d c}\left[S_{1}, S_{2}, S_{3}\right]^{T}$. The components, i.e. switch position of the converter, are generated by a look up table of FPGA controller (Table I and Fig. 3).

\section{Discrete-event Current Control}

To consider a hysteresis controller as a discrete-event dynamical system, it allows the formation in a much higher detail on the switching actions, and will enable a better understanding of the controller design [4]. A discrete-event system reacts only if an event is recognized [10].

\begin{tabular}{|c|c|c|c|c|c|c|c|}
\hline & $\mathrm{Su} 1$ & $\mathrm{Su} 2$ & $\mathrm{Su} 3$ & $\mathrm{Su} 4$ & Su5 & Su6 \\
\hline & & 100 & 110 & 010 & 011 & 001 & 101 \\
\hline \begin{tabular}{|l|} 
Sdi0 \\
\end{tabular} & 000 & V7 & V0 & V7 & V0 & V7 & V0 \\
\hline Sdi1 & 100 & V1 & V1 & V7 & V0 & V7 & V1 \\
\hline Sdi2 & 110 & V2 & V2 & V2 & V0 & V7 & V0 \\
\hline Sdi3 & 010 & V7 & V3 & V3 & V3 & V7 & V0 \\
\hline Sdi4 & 011 & V7 & V0 & V4 & V4 & V4 & V0 \\
\hline \begin{tabular}{|l|} 
Sdi5 \\
\end{tabular} & 001 & V7 & V0 & V7 & V5 & V5 & V5 \\
\hline Sdi6 & 101 & V6 & V0 & V7 & V0 & V6 & V6 \\
\hline \begin{tabular}{|l|l} 
Sdi7 \\
\end{tabular} & 111 & V7 & V0 & V7 & V0 & V7 & V0 \\
\hline
\end{tabular}

Table I. - Look-up Table

To control the current $\boldsymbol{i}_{s}$, the sector of the grid voltage $\boldsymbol{e}_{s}$ is recognized first. Based on the known sector, the input voltage vector $\boldsymbol{u}_{S}\left(\boldsymbol{V}_{i}\right)$ (the transistor switching pattern) for the current control is selected in respect to the current control error, related to Lyapunov stability condition. To consider a space vector representation of the grid voltage $\boldsymbol{e}_{s}$, the voltage is represented as vector that rotates around the origin. Six active switching vectors of the three-phase transistor converter result in six active input voltage vectors denoted $\boldsymbol{V}_{1} \ldots \boldsymbol{V}_{6} ; \boldsymbol{V}_{0}$ and $V_{7}$ are two zero voltage vectors. According to signs of the phase voltages $u_{s 1}, u_{s 2}$ and $u_{s 3}$, the phase plane is divided into six sectors denoted Su1 ... Su6, Fig. 3 .

In regards to the situation, the grid voltage space vector $\boldsymbol{e}_{s}$ is in sector 1 . In this sector, voltage vectors $\boldsymbol{V}_{0}, \boldsymbol{V}_{1}$, $\boldsymbol{V}_{2}, \boldsymbol{V}_{6}$ and $\boldsymbol{V}_{7}$ are selected for the current control. $\boldsymbol{V}_{0}$, $\boldsymbol{V}_{7}$ are two zero vectors, while $\boldsymbol{V}_{1}, \boldsymbol{V}_{2}, \boldsymbol{V}_{6}$ are three nearest adjacent live input voltage vectors to this sector. With the use of the discrete event system theory, five input voltage vectors $\boldsymbol{V}_{0}, \boldsymbol{V}_{1}, \boldsymbol{V}_{2}, \boldsymbol{V}_{6}$ and $\boldsymbol{V}_{7}$ are recognized as discrete states of the system. Events represent the allowed transition among the discrete states i.e. allowed switching. The structure of the proposed strategy is represented by Petri Net graph in Fig. 4.

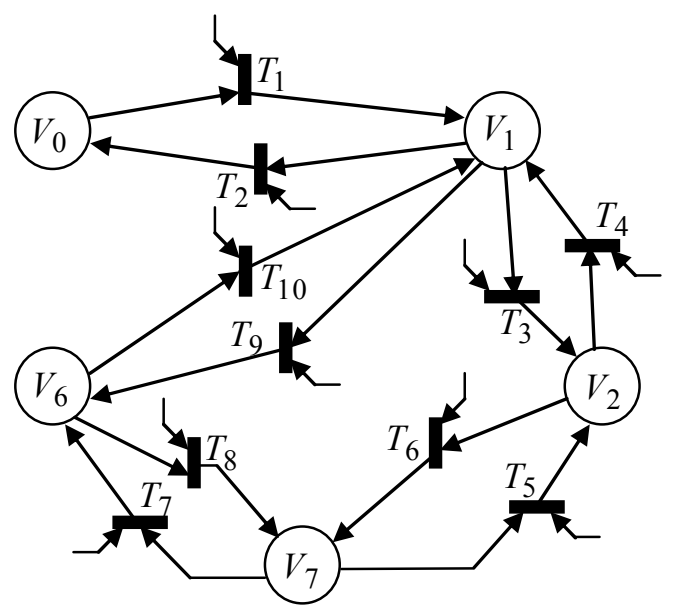

Fig. 4. PN-graph of the switching sequence in Sector 1 
The Petri net formalism is propriety for research on possible deadlock or livelock of discrete event system under study [10]. Switching among the available input voltage vectors in each sector is determined by the conditions that originate from the derivative of the Lyapunov function.

\section{Lyapunov-based Control Strategy}

The analysis of the three-phase boost rectifier is a very challenging theoretical problem because we add to the complexity, of controlling a nonlinear multivariable uncertain system, the fact that the control signal lives in finite set $\boldsymbol{S}\left(S_{1}, S_{2}, S_{3}, \bar{S}_{1}, \bar{S}_{2}, \bar{S}_{3}\right)$, hence we are confronted with the hybrid system.

The aim of this paper is the design of a control law for a boost rectifier in order to achieve a good output voltage and grid current control in a steady state and transient operation conditions. The feedback system is globally asymptotically stable in the sense of Lyapunov stability theory. Therefore, we are interested in the aim extension of the Lyapunov function concepts.

On the basis of Lyapunov stability theory, a positive definite scalar function $V$, as a candidate for the Lyapunov function, is to be found, such that the total energy of the system is continuously dissipated. In such a case, any nonlinear system must eventually settle down to an equilibrium point.

For the Lyapunov function candidate

$$
V=(1 / 2) \Delta \boldsymbol{i}_{\mathbf{s}}^{T} \Delta \boldsymbol{i}_{\mathbf{s}}=(1 / 2)\left(\boldsymbol{i}_{s}-\boldsymbol{i}_{s}^{d}\right)^{T}\left(\boldsymbol{i}_{s}-\boldsymbol{i}_{s}^{d}\right),
$$

the stability requirement will be fulfilled if control can be selected as such, that the derivative of the Lyapunov function candidate is negative $\dot{V}=\Delta \boldsymbol{i}_{S}{ }^{T} \Delta \dot{\boldsymbol{i}}_{s} \leq 0$. Derivatives of the current control error (6) may be expressed with the voltage equation

$$
(d / d t)\left(\boldsymbol{i}_{s}-\boldsymbol{i}_{s}^{d}\right)=(1 / L)\left(\boldsymbol{e}_{s}-R \boldsymbol{i}_{s}-\boldsymbol{u}_{s}\left(\boldsymbol{V}_{i}\right)\right)-(d / d t) \boldsymbol{i}_{s}^{d},
$$

where $\boldsymbol{i}_{s}^{d}, \boldsymbol{i}_{s}$ are desired and actual grid current, $\boldsymbol{u}_{s}\left(\boldsymbol{V}_{i}\right)$ is voltage control input, $R \boldsymbol{i}_{S}$ is resistive voltage drop and $\boldsymbol{e}_{s}$ is ac input voltage.

For $d\left(\Delta \boldsymbol{i}_{s}\right) / d t=0$ the equivalent control voltage [11] can be expressed as:

$$
u_{e q u}=\boldsymbol{e}_{s}-R \boldsymbol{i}_{s}-L(d / d t) \boldsymbol{i}_{s}^{d}
$$

and derivative of Lyapunov function is:

$$
\dot{V}=\left(\boldsymbol{i}_{s}-\boldsymbol{i}_{s}^{d}\right)\left(u_{e q u}-\boldsymbol{u}_{s}\left(\boldsymbol{V}_{i}\right)\right) / L<0
$$

The conditions for the sequential switching of the power converter are selected as:

$$
\begin{aligned}
& S_{1}=(1 / 2)(1-\operatorname{sign}(A)), S_{2}=(1 / 2)(1-\operatorname{sign}(B)), \\
& S_{3}=(1 / 2)(1-\operatorname{sign}(C))
\end{aligned}
$$

$$
\begin{aligned}
& A=\left(i_{s a}-i_{s a}^{d}\right) \\
& B=-(1 / 2)\left(i_{s a}-i_{s a}^{d}\right)-(\sqrt{3} / 2)\left(i_{s b}-i_{s b}^{d}\right) \\
& C=-(1 / 2)\left(i_{s a}-i_{s a}^{d}\right)+(\sqrt{3} / 2)\left(i_{s b}-i_{s b}^{d}\right)
\end{aligned}
$$

which is evolved from the Lyapunov function derivative. When $U_{d c}$ has enough magnitude that $\dot{V} \leq 0$, than $V \rightarrow 0$ and $\boldsymbol{i}_{s} \rightarrow \boldsymbol{i}_{s}^{d} . S_{1}, S_{2}$ and $S_{3}$ represent the switching state of the three-phase power converter. Notice that if $S_{l}$, $S_{2}, S_{3}$ equal to zero simultaneously, no current is delivered to the dc load.

The proposed logical event-driven grid current control can be realized in the form described in Table I, where states of grid current control error are presented by $\operatorname{sign}\left(D_{j}\right)\left(D_{j}=S_{1}, S_{2}, S_{3}\right)$ and currently active voltage sector is presented by sign $E_{S}\left(e_{S 1}, e_{S 2}, e_{S 3}\right)$. To further improve the presentation, active voltage vectors are marked in Table I with a blue background. Because the transition between converter switch states is performed by switching only one converter leg, converter switching frequency grid current chattering are reduced.

\section{E. Discretized Output Voltage Control}

The discretized dc output voltage control will be designed for the control system expressed by (2). The unity power factor of ac/dc boost rectifier will be achieved for $u_{C}=u_{d}, u_{q}=0$ in $\mathrm{d}$-q output reference frame.

The output voltage control error

$$
\sigma_{u}=u_{C}^{d}-u_{C}
$$

determines the input dc current $i_{d c}$ which also plays the role of the rectifier output current. With $\dot{\sigma}_{u}=-d_{u} \sigma_{u}$ we get a robust PI-control algorithm [8]:

$$
i_{d c}(k)=i_{d c}(k-1)+\frac{G_{u} C}{T}\left(\left(1+T d_{u}\right) \sigma_{u}(k)-\sigma_{u}(k-1)\right) .
$$

In the proposed algorithm the output load resistance $R_{L}$ does not appear, therefore the control is independent of its value. The resistive load can change its value in transient states, i.e. which includes step change of its value. The rectifier dc current $i_{d c}$, which forms an input for $\mathrm{dc}$ boost rectifier part is influenced by a dc output voltage error $\sigma_{u}(k)$, dc bus capacitor $C$, used sampling time $T$ and feedback gain $G_{u}$. The asymptotical stability of algorithm is guaranteed with the proper choice of parameter $d_{u}$.

The reference input active power would be determined with dc current $i_{d c}$ and bus voltage $U_{d c}$ :

$$
p^{d}=U_{d c} i_{d c}
$$

In Fig. 5, the complete block diagram of proposed discretized output voltage and ac input current control scheme in hybrid switching control is presented.

where 


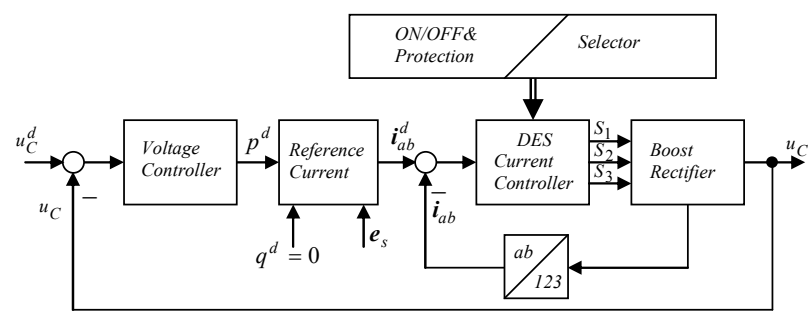

Fig. 5. Proposed control scheme

\section{Unity Power Factor}

In the proposed nonlinear control scheme, which has an ability to obtain sinusoidal shaped ac input current, we are going to profit from the benefits of robust switching control. These benefits are insensitive to parameter variations and excellent decoupling properties. The sinusoidal form of ac currents is assured with the proposed discrete event current control. The actual current, tracks the desired value without a delay, it is robust to the variations of resistance $R$ and inductance $L$ and it decouples the influences between grid phases. We assume that the grid frequency remains constant. The time constant that determines the transient of ac input currents $T_{s}=L / R$ is some order smaller than the bulky designed dc time constant $T_{d c}=R_{L} C$, which determines the slow change of output dc voltage $u_{C}$. This physical property of time dependent variability of ac input current and slow change of output dc voltage can be profitably used to express the relation between the input ac power and the dissipated output dc power.

The calculation of active and reactive components of modulating currents is produced by the method of instantaneous power, known as the "Akagi-Nabae theory" [12]. This theory, also known as pq-theory, is based on Clark transformation, i.e. 123/ab. Thus, the reference current signals $i_{s a}^{d}, i_{s b}^{d}$ can be received as:

$$
\left[\begin{array}{c}
i_{s a}^{d} \\
i_{s b}^{d}
\end{array}\right]=\frac{1}{e_{s a}^{2}+e_{s b}^{2}}\left[\begin{array}{cc}
e_{s a} & e_{s b} \\
e_{s b} & -e_{s a}
\end{array}\right]\left[\begin{array}{c}
p^{d} \\
q^{d}
\end{array}\right]
$$

where $p^{d}$ and $q^{d}$ are reference active and reactive power signals. Reference current signals in three-phase system are calculated by reverse Clark transformation.

For unity power factor $q^{d}=0$ and the reference grid current will be:

$$
i_{s a}^{d}=\frac{e_{s a}}{e_{s a}^{2}+e_{s b}^{2}} p^{d}, \quad i_{s b}^{d}=\frac{e_{s b}}{e_{s a}^{2}+e_{s b}^{2}} p^{d}
$$

One of the main advantages of pq-theory is that there is no need for phase synchronization with system voltages. Also, average components $\mathrm{p}$ and $\mathrm{q}$ can be easily detected (filtered) by means of low-order low-pass filters. The latter makes positive impact on system dynamic and stability. The converter control system block diagram is shown in Fig. 5.

\section{Implementation}

The proposed approach is based on fast parallel processing and is suitable for a Field Programmable Gate Array (FPGA) implementation [13]. In such implementation, it would be possible to reproduce near ideal switching mode process. However, with FPGA implementation, designer has a difficult task to characterize and describe the hardware architecture that corresponds to the chosen control algorithm. FPGA designers must follow an efficient design methodology in order to benefit from the advantages of the FPGAs and their powerful CAD tools. From a software point of view, HDL modeling system is based on the use of variables that request logic values, too.

FPGA implementation of Table I is presented in Fig. 6, where sign $D_{1}$, sign $D_{2}$ and sign $D_{3}$ present $S_{1}, S_{2}$ and $S_{3}$, respectively. Voltage sector states are presented by $\operatorname{sign}\left(e_{S I}\right), \operatorname{sign}\left(e_{S 2}\right), \operatorname{sign}\left(e_{S 3}\right)$. Converter leg switching outputs are denoted as follows: TOP1 and BOT1 present states of top and bottom transistor in converter leg $S_{1}, \bar{S}_{1}$, TOP2 and BOT2 present states of transistors in converter leg $S_{2}, \bar{S}_{2}$, whereas TOP3 and BOT3 present states of transistors in converter leg $S_{3}, \bar{S}_{3}$.

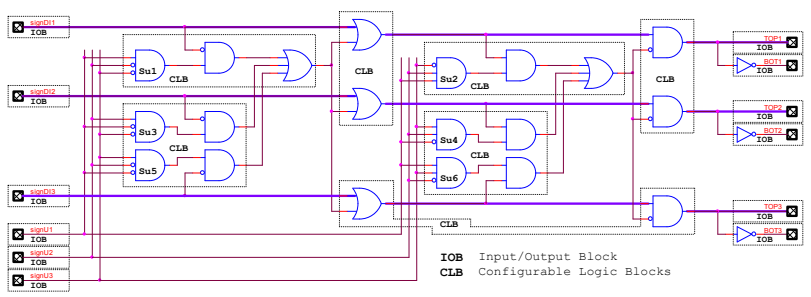

Fig. 6: FPGA schematic circuits

\section{Results}

The discrete event current control algorithm was implemented on the in-house developed DSP/FPGA board [14]. The DSP/FPGA board contains Texas Instruments TMS 320C32 digital signal processor and Xilinx Spartan family field programmable gate array. DSP serves for $A / D$ conversion and to generate the reference current. By replacing the usual sequential calculation of algorithms on the DSP, by parallel executable FPGA, the hardware increases the calculation speed. A/D conversion is the most critical operation in regards to time and takes $5 \mu \mathrm{s}$. According to the fact that $\mathrm{A} / \mathrm{D}$ conversion takes most of the calculation time, to switch frequencies up to $200 \mathrm{kHz}$ is theoretically possible.

Fig. 7 shows some simulation results. L-R filter on ac side has the inductance value $L=5 \mathrm{mH}$ and resistance $R$ $=1 \Omega$. Load resistance on the dc side has nominal value $R_{L}=22 \Omega$ and was changed during the simulation time. Capacitance $C=4,5 \mathrm{mF}$. Load step change from $110 \Omega$ to $22 \Omega$ was made at time $0.12 \mathrm{~s}$. The sampling time interval is $20 \mu \mathrm{s}$. 

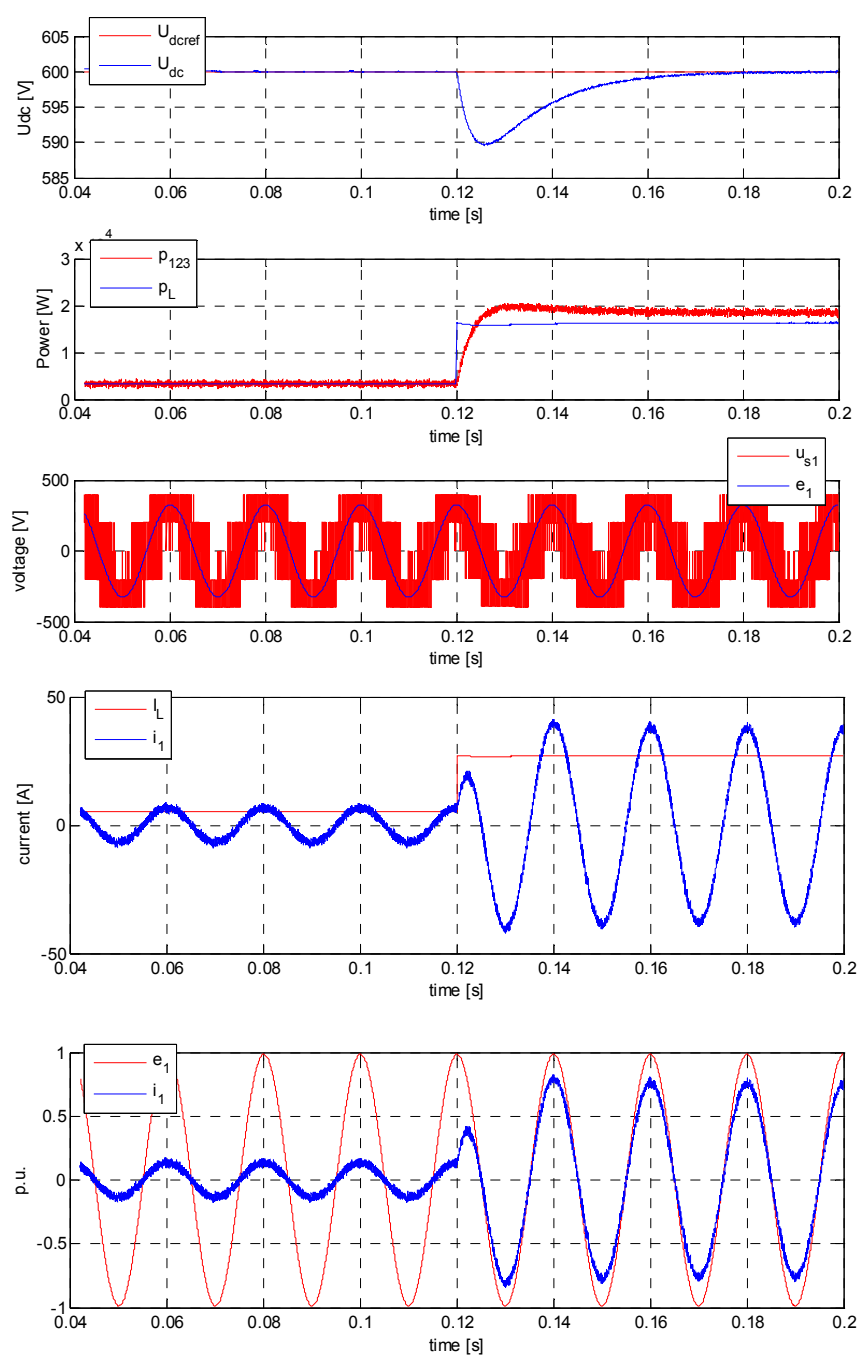

Fig. 7. Change in load at time $0.12 \mathrm{~s}$. From top to bottom: $\mathrm{U}_{\mathrm{dcref}}$ - reference dc bus voltage, $\mathrm{U}_{\mathrm{dc}}-$ actual dc bus voltage, $\mathrm{p}_{123}$ - input power, $\mathrm{p}_{\mathrm{L}}$ - power on load $R_{\mathrm{L}}, \mathrm{u}_{\mathrm{s} 1}$ - voltage on first leg of converter, $\mathrm{u}_{1}-$ input voltage in phase $1, \mathrm{i}_{\mathrm{L}}-$ load current, $i_{1}$ - input current in phase 1 .

\section{Conclusion}

The idea of a hybrid based control of event driven systems is used for the design of an event-driven current controller and auxiliary steering and protection functions for a three phase rectifier. Individual control functions have been designed with the use of the proposed approach, and integrated into the overall functionality description of the converter. Overall functionality of the boost rectifier and the performance of the proposed event driven current control were checked by simulations and experimentally confirmed. Special attention is paid to the mapping of the proposed design approach into the schematic form for the FPGA implementation. The simulation and experiment confirmed potentials of presented approach: traditional coding efforts are significantly reduced on one hand, and on the other hand the control algorithm can be verified off-line.

In this study, the active power control problem of boost rectifier was addressed. The nonlinear behavior of the system limits the performance of chosen hysteresis controllers used for this purpose. This paper has successfully demonstrated the design, stability analysis and simulation and tests of a Lyapunov technique approach. The feedback system is globally asymptotically stable in the sense of Lyapunov method. The transient state and steady state performances of the logical based controller were improved via Lyapunov stability. Consequently, a high performance dynamic, despite the presence of parameter uncertainties or disturbances, is obtained.

\section{References}

[1] H.M. Suryawanshi, M.R. Ramteke, K.L. Thakre and V.B. Borghate, "Unity-Power-Factor Operation of Three-Phase AC-DC Soft Switched Converter based on Boost Active Clamp Topoly in Modular Approach". IEEE Tran. Power Electronics, vol. 23, no. 1, pp. 229-236, 2008.

[2] J. Dannehl and F.W. Fuchs, "Discrete Sliding Mode Current Control of Three-phase Grid-Connected PWM Converters". In Proc. $13^{\text {th }}$ European Conference on Power Electronics and Applications, EPE'09, pages 1-10, 2009.

[3] A. Tilli A and A. Tonielli, "Sequential Design of Hysteresis Current Controller for Three-Phase Inverter". IEEE Trans. on Industrial Electronics, vol. 45, no. 5, pp. 771-781, 1998.

[4] A. Polic and K. Jezernik, "Closed-loop matrix based model of discrete event systems for machine logic control design". IEEE Trans. Industrial Informatics, vol. 1, no. 1, pp. 39-46, 2005.

[5] M.S. Branicky, V.S. Borkar and S.K. Mitter, "A unified framework for hybrid control: model and optimal control theory," IEEE Trans. Autom. Control., vol. 43, no. 1, pp. 31-45, Jan. 1998.

[6] M.N. Cirstea, "Modeling Environment for Power Electronic Systems Integrated Development and Controller Prototyping". Proc. of IEEE ISIE 2005, Dubrovnik, Croatia, 2005.

[7] P. Antoniewicz and M.P.Kazmierkowski, "Virtual-FluxBased Predictive Direct Power Control of AC/DC Converters With Online Inductance Estimation". IEEE Tran. Ind. Electronics, vol. 55, no. 12, pp. 4381 - 4390, 2008.

[8] A. Sabanovic, K. Jezernik and N. Sabanovic, "Sliding Modes Applications in Power Electronics and Electrical Drives". In Lecture notes in control and information sciences, 1st ed., Y.U. Xinghuo, X.U. Jian-Xin, (Ed.). Berlin: Springer, pp. 223-251, 2002.

[9] P.J.G. Ramadge and W.M. Wonham, "The Control of Discrete Event Systems". Proceedings of the IEEE, vol.77, no. 1, pp. 81-99, 1989.

[10] L.E. Holloway, B.H. Krogh and A. Giua, "A Survey of Petri Net Methods for Controlled Discrete Event Systems". Journal of Discrete Event Dynamical Systems: Theory and Applications, vol. 7, no. 2, pp. 151-190, 1997.

[11] V.I. Utkin, Sliding Modes in Control and Optimization. Berlin: Springer Verlag, 11992.

[12] H. Akagi, Y. Kanazava and A. Nabae, "Generalized Theory of the Instantenous reactive Power in Three-phase Circuits". In Proc. of the 1983 Int. Power Electronics Conference, Tokyo, Japan, 1983.

[13] E. Monmasson and M.N. Cirstea, "FPGA Design Methodology for Industrial Control Systems - A Review". IEEE Tran. Ind. Electronics, vol. 54, no. 4, pp. 1824-1842, 2007.

[14] D. Hercog, M. Čurkovič, G. Edelbaher and E. Urlep, "Programming of the DSP2 board with the Matlab/Simulink". In Proc. of IEEE ICIT 2003, Maribor, Slovenia, pp. 709-713, 2003. 